\title{
Lindner, Martin
}

\section{Werkstattarbeit in der Biologiedidaktik. Umstellung eines Praktikums auf Projektarbeit}

Kramer, Kathrin [Hrsg.]; Rumpf, Dietlinde [Hrsg.]; Schöps, Miriam [Hrsg.]; Winter, Stephanie [Hrsg.]: Hochschullernwerkstätten - Elemente von Hochschulentwicklung? Ein Rückblick auf 15 Jahre Hochschullernwerkstatt in Halle und andernorts. Bad Heilbrunn : Verlag Julius Klinkhardt 2020, S. 379-395. - (Lernen und Studieren in Lernwerkstätten)

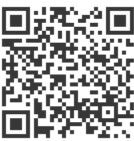

Quellenangabe/ Reference:

Lindner, Martin: Werkstattarbeit in der Biologiedidaktik. Umstellung eines Praktikums auf Projektarbeit In: Kramer, Kathrin [Hrsg.]; Rumpf, Dietlinde [Hrsg.]; Schöps, Miriam [Hrsg.]; Winter, Stephanie [Hrsg.]: Hochschullernwerkstätten - Elemente von Hochschulentwicklung? Ein Rückblick auf 15 Jahre Hochschullernwerkstatt in Halle und andernorts. Bad Heilbrunn : Verlag Julius Klinkhardt 2020, S. 379-395 - URN: urn:nbn:de:0111-pedocs-212292 - DOI: 10.25656/01:21229

https://nbn-resolving.org/urn:nbn:de:0111-pedocs-212292

https://doi.org/10.25656/01:21229

in Kooperation mit / in cooperation with:

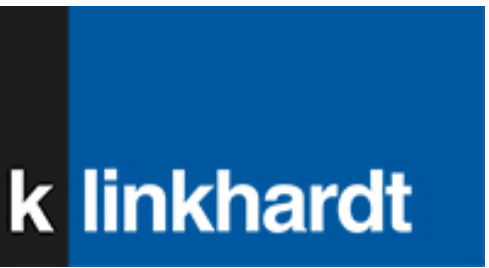

http://www.klinkhardt.de

\section{Nutzungsbedingungen}

Dieses Dokument steht unter folgender Creative Commons-Lizenz: http://creativecommons.org/licenses/by-nc-sa/4.0/deed.de - Sie dürfen das Werk bzw. den Inhalt unter folgenden Bedingungen vervielfältigen, verbreiten und öffentlich zugänglich machen sowie Abwandlungen und Bearbeitungen des Werkes bzw. Inhaltes anfertigen: Sie müssen den Namen des Autors/Rechteinhabers in der von ihm festgelegten Weise nennen. Dieses Werk bzw. der Inhalt darf nicht für kommerzielle Zwecke verwendet werden. Die neu entstandenen Werke bzw. Inhalte dürfen nur unter Verwendung von Lizenzbedingungen weitergegeben werden, die mit denen dieses Lizenzvertrages identisch oder vergleichbar sind.

Mit der Verwendung dieses Dokuments erkennen Sie die Nutzungsbedingungen an.

\section{Terms of use}

This document is published under following Creative Commons-License: http://creativecommons.org/licenses/by-nc-sa/4.0/deed.en - You may copy, distribute and transmit, adapt or exhibit the work in the public and alter, transform or change this work as long as you attribute the work in the manner specified by the author or licensor. You are not allowed to make commercial use of the work. If you alter, transform, or change this work in any way, you may distribute the resulting work only under this or a comparable license.

By using this particular document, you accept the above-stated conditions of use.

\section{Kontakt / Contact:}

\section{peDOCS}

DIPF | Leibniz-Institut für Bildungsforschung und Bildungsinformation Informationszentrum (IZ) Bildung

E-Mail: pedocs@dipf.de

Internet: www.pedocs.de

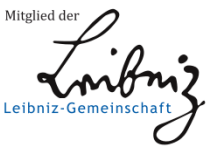




\section{Lernen und Studieren in Lernwerkstätten}

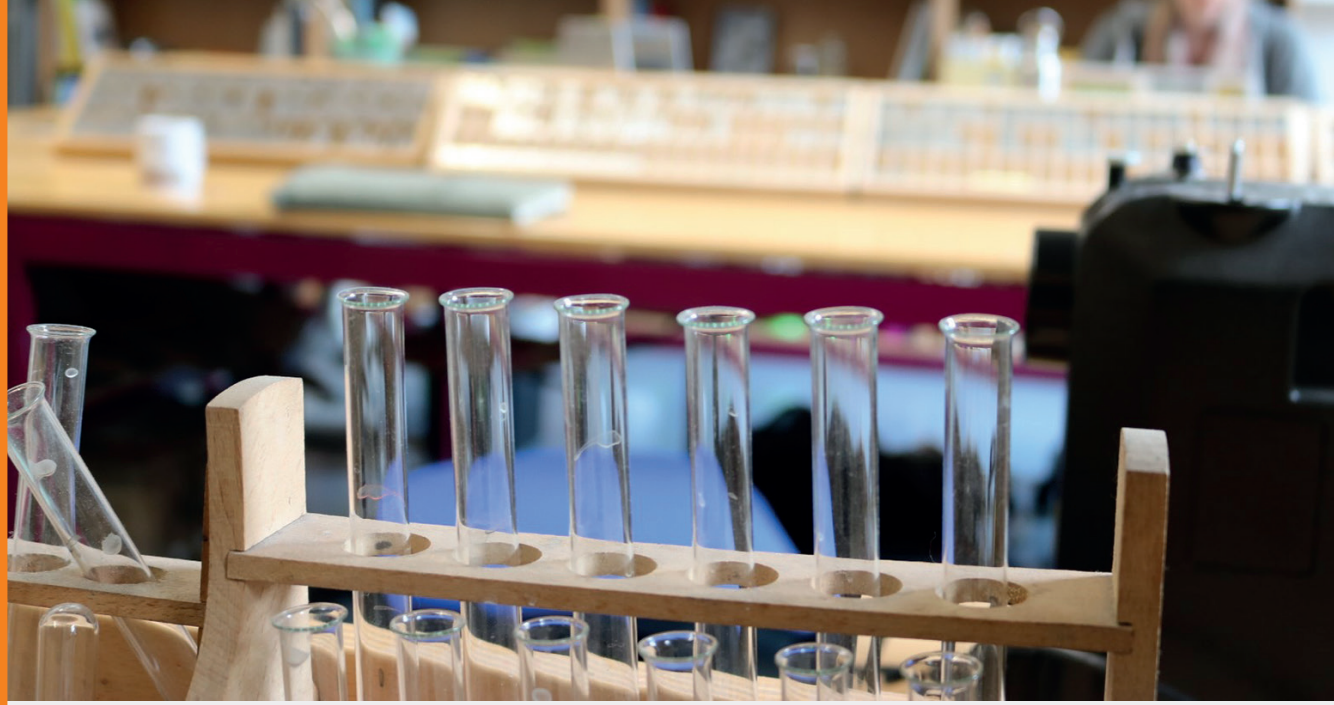

Kathrin Kramer / Dietlinde Rumpf / Miriam Schöps / Stephanie Winter (Hrsg.)

Hochschullernwerkstätten - Elemente von Hochschulentwicklung?

Ein Rückblick auf 15 Jahre Hochschullernwerkstatt in Halle und andernorts 


\section{Kramer / Rumpf / Schöps / Winter Hochschullernwerkstätten - Elemente von Hochschulentwicklung?}




\section{Lernen und Studieren in Lernwerkstätten}

Impulse für Theorie und Praxis

Herausgegeben von

Eva-Kristina Franz, Johannes Gunzenreiner, Barbara Müller-Naendrup, Hartmut Wedekind und Markus Peschel 


\section{Kathrin Kramer \\ Dietlinde Rumpf \\ Miriam Schöps \\ Stephanie Winter \\ (Hrsg.)}

\section{Hochschullernwerkstätten - Elemente von Hochschulentwicklung?}

Ein Rückblick auf 15 Jahre Hochschullernwerkstatt in Halle und andernorts 
Der vorliegende Band ist anlässlich einer Tagung des 15jährigen Bestehens der Hochschullernwerkstatt Erziehungswissenschaften in Halle im November 2020 entstanden.

Gefördert wurden die Tagung und der Band vom Zentrum für Lehrer *innenbildung, der Universitäts- und Landesbibliothek Sachsen-Anhalt sowie der Martin-Luther-Universität Halle-Wittenberg.

Dieser Titel wurde in das Programm des Verlages mittels eines Peer-Review-Verfahrens aufgenommen. Für weitere Informationen siehe www.klinkhardt.de.

Bibliografische Information der Deutschen Nationalbibliothek Die Deutsche Nationalbibliothek verzeichnet diese Publikation in der Deutschen Nationalbibliografie; detaillierte bibliografische Daten sind im Internet abrufbar über http://dnb.d-nb.de.

2020.k. (C) by Julius Klinkhardt.

Coverfoto: (C) Florian Johnke-Liese / Hochschullernwerkstatt.

Druck und Bindung: AZ Druck und Datentechnik, Kempten.

Printed in Germany 2020.

Gedruckt auf chlorfrei gebleichtem alterungsbeständigem Papier.

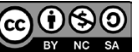

Die Publikation (mit Ausnahme aller Fotos, Grafiken und Abbildungen) ist veröffentlicht unter der Creative Commons-Lizenz: CC BY-NC-SA 4.0 International https://creativecommons.org/licenses/by-nc-sa/4.0/ 


\section{Inhalt}

Kathrin Kramer, Dietlinde Rumpf, Miriam Schöps und Stephanie Winter Einführung in den Band

\section{Teil 1: Historische Entwicklung}

Miriam Schöps und Dietlinde Rumpf

Universität mit Lernwerkstatt in Entwicklung -

Von der Materialausleihe zur Hochschuldidaktik

Hartmut Wenzel

Lernwerkstätten auch für die Sekundarstufen -

Zur Entwicklung der Hochschullernwerkstatt in Halle

Ulrike Stadler-Altmann, Susanne Schumacher, Enrico A. Emili,

Gerda Winkler und Elisabeth Dalla Torre

Hochschullernwerkstätten als Spielball der Bildungspolitik?

Die EduSpace Lernwerkstatt in der Südtiroler Lehrer*innenbildung zwischen nationalen und regionalen Bildungsinteressen

Hartmut Wedekind und Corinna Schmude

Von der Idee zum strukturell verankerten hochschuldidaktischen Prinzip -

Lernwerkstätten und Lernwerkstattarbeit im Studiengang „Erziehung und Bildung in der Kindheit" an der Alice Salomon Hochschule Berlin 68

\section{Teil 2: Wahrnehmung, Selbstverständnis, Einflüsse}

Dietlinde Rumpf und Corinna Schmude

NeHle - Internationales Netzwerk der Hochschullernwerkstätten -

Entwicklungsphasen einer Interessenvertretung und

eines gemeinsamen Begriffsverständnisses

Constantin Beyer und Florian Johnke-Liese

Hochschullernwerkstatt goes digital!

Alexandra und Michael Ritter

Drucken wie ,von gestern' in der Schule der Zukunft -

Die Schuldruckerei als Erfahrungsraum für Kinder und Studierende 
Livia Enders (geb. Makrinus) und Stephanie Winter

Studierende und Dozierende im Spannungsfeld zwischen

Theorie und Praxis - Die Hochschullernwerkstatt als Forschungsraum

John Marcus Sommer

Selbstwahrnehmung in der Hochschullernwerkstatt -

Wissenschaft zwischen Abstraktion und emotionalem Lernen

Lena Kliebe

Hochschullernwerkstatt als Raum und pädagogische Konzeption -

„Mein eigener Lernprozess“

Jerome Kampe

Geschichtsunterricht in der Lernwerkstatt!?

\section{Teil 3: Partizipation und Inklusion}

Melanie Schlag

Hochschullernwerkstatt schlägt Schlager

Kathrin Kramer

Nichtreformistische Reformen - Die Bedeutung von

Hochschullernwerkstätten auf dem Weg zu einer inklusiven Universität am Beispiel des Budgets für Arbeit

Johanna Ingenerf und Pascal Kurz

Menschenrechtsbasierte Bildung im internationalen Dialog -

Ein selbstorganisiertes Seminar mit Austausch

Ines Boban und Andreas Hinz

Hochschullernwerkstatt Halle als Möglichkeitsort -

Von WIRkstatt-Impulsen für inklusive Prozesse

Brigitte Kottmann und Alena Lensker (geb. Beckmann)

Die Lernwerkstatt und der Studiengang „Integrierte Sonderpädagogik“

an der Universität Bielefeld: Impulse, Synergien und Reflexionen

Sarah Dannemann, Tjark Neugebauer, Claudia Schomaker und Rolf Werning

Die LeibnizLernlandschaft: Diversität und Digitalisierung $\left(\mathrm{L}^{2} \mathrm{D}^{2}\right)$

gestalten - Konzeptionelle Gedanken für eine inklusive

Hochschullernwerkstatt an der Leibniz Universität Hannover 


\section{Teil 4: Reflexivität und Professionalisierung}

Kira Wybierek

Praxissemester in der Hochschullernwerkstatt -

Reflexion eines Lernprozesses

Eva Hoffart

„...da man lernt, eigene Gedanken und Ideen noch einmal zu vertiefen“ -

Theoretische Überlegungen und praktische Umsetzungen zum Reflektieren

von Lehramtsstudierenden

Edita Jung und Lena S. Kaiser

„Vielleicht romantisiere ich die Räume meiner Kindheit.“

Zugänge zu einer biographischen Reflexivität in

kindheitspädagogischen Hochschullernwerkstätten

Rolf-Torsten Kramer

Zum Problem der Professionalisierung im Lehramtsstudium und

zum Potenzial der Hochschullernwerkstatt

Mark Weißhaupt, Kathleen Panitz und Elke Hildebrandt

Die Inszenierung von „Theorie und Praxis“ sowie

„Neugier und Desinteresse“ bei der Professionalisierung

angehender Lehrpersonen in Hochschullernwerkstätten

Markus Peschel und Pascal Kihm

Hochschullernwerkstätten - Rollen, Rollenverständnisse und

Rollenaushandlungen

David Paulus, Patrick Gollub und Marcel Veber

Forschendes Lernen und Kasuistik: Überschneidungen und

Abgrenzungen bezogen auf Reflexivität in der

Hochschullernwerkstattarbeit

Pascal Kihm, Jenny Diener und Markus Peschel

Qualifizierungsprozesse und Qualifikationsarbeiten in

Hochschullernwerkstätten - Forschende Entwicklung einer

innovativen Didaktik 
10| Inhaltsverzeichnis

\section{Teil 5: Kooperation}

Georg Breidenstein, Sara Burkhardt, Thorid Rabe und Miriam Schöps

Zur Materialität des Lernens -

Anregungen aus einem interdisziplinären Forum

in der Hochschullernwerkstatt

Miriam Asmus, Kati Friebe, Mirjam Lewin und Kati Misselwitz

Entdeckendes Lernen und Digitale Medien - Ein Traumpaar .

Siglinde Spuller

Kooperation und Kooperatives Lernen als Prinzip

Hochschullernwerkstätten-adäquaten Lernens?

Eine konzeptionelle Verortung

Linda Balzer

Der Effekt selbstregulierenden und forschenden Lernens auf kooperative und individuelle Lernprozesse in der

Lernwerkstatt Religion Plural (LeRP)

Martin Lindner

Werkstattarbeit in der Biologiedidaktik -

Umstellung eines Praktikums auf Projektarbeit

Autor*innenbeschreibungen 
Martin Lindner

\section{Werkstattarbeit in der Biologiedidaktik - Umstellung eines Praktikums auf Projektarbeit}

\section{Zusammenfassung}

Das klassische Seminar der Biologiedidaktik an der Martin-Luther-Universität Halle-Wittenberg wurde vor 10 Jahren auf (Lern-)Werkstattarbeit umgestellt. In einem Raum, der als offene Werkstatt hergerichtet wurde, sind Praktikumsmaterial, W-Lan, Laptops, ein interaktiver Screen und Bücher vorhanden, im benachbarten Labor können Experimente durchgeführt werden. Der Raum steht den Studierenden an drei Tagen mit Betreuung durch eine Laborantin und mindestens einer Person aus dem wissenschaftlichen Personal zur Verfügung, darüber hinaus können sich die Studierenden auch zu anderen Zeiten dort treffen. Die Arbeit wird in studentischen Projekten organisiert. Jeweils zwei oder drei Studierende arbeiten zusammen, um im Laufe des Semesters ein oder zwei Unterrichtsprojekte zu erstellen. In diesem Artikel wird zunächst begründet, warum diese Form für das Lehramtsstudium gewählt wurde. Im Anschluss daran werden einige Ergebnisse vorgestellt und schließlich Daten aus der Auswertung des ersten Semesterdurchganges durch diese Form des Studiums dargestellt.

\section{Begründung für die Einführung von Werkstattarbeit in Projekten}

Die nötigen Veränderungen für die Schulen der Zukunft sind nur durch eine Offenheit gegenüber Innovationen möglich und nur in Teamarbeit umsetzbar. Die Fachgruppe in der Schule wurde verschiedentlich als Nukleus für Schulentwicklung identifiziert. Damit Lehrkräfte in einer offenen Haltung - offen für Neuerungen, offen für die Arbeit im (Fach-)Team und erfahren in der Umsetzung von Innovationsvorhaben - an die Arbeit gehen können, müssen sie sich bereits im Studium mit solchen Aufgaben befassen. Die Kultusministerkonferenz (KMK) hat dies in ihren Standards für die Lehrer*innenbildung als Anforderung formuliert. Nach der Präambel der Standards in Bezug auf die Weiterentwicklung der Schule wird als Kompetenz 11 genannt: „Lehrerinnen und Lehrer beteiligen sich an der Planung und Umsetzung schulischer Projekte und Vorhaben" (KMK 2004, 13). Wir verstehen Kompetenz dabei im Weinertschen Sinne als eine um- 
fassende Haltung, die neben Wissen vor allem Können, aber auch die Bereitschaft zur Umsetzung beinhaltet (FISCHLER et al. 2018).

Die Beteiligung von Studierenden an Projekten ist eine weit verbreitete Praxis, die beispielsweise in den Wirtschaftswissenschaften und Ingenieurstudiengängen sowie in Management-Seminaren genutzt werden. Hier sind sie vor allem deshalb von Vorteil, weil die Absolvent*innen sich auf komplexe Situationen vorbereiten, die in ihrer Vielfältigkeit und ihrer Unübersichtlichkeit nicht mit eindeutigen und in einem Studium gelernten Lösungen zu meistern sind, sondern eine an die jeweilige Situation angepasste Lösung verlangen. Hierzu benötigt es umfassende und flexible Kompetenzen im persönlichen und im professionellen Bereich, die in Übungssituationen erworben wurden.

Wodurch unterscheidet sich aber Schule und schulisches Handeln von solchen Situationen, in denen sich Manager bewähren müssen? Der Schulalltag ist von zahlreichen unplanbaren Faktoren bestimmt, so dass Management eigentlich eine angemessene Beschreibung von Lehrer*innenhandeln ist. Allein das Zeitmanagement des Lehrer*innenberufes mag als Beispiel dafür dienen, wie dynamisch und auch unvorhersehbar das Berufsfeld ist. Dabei ist neben dem optimierten Zeitmanagement im Unterricht (HeLmKe 2017) natürlich auch ein professionelles Management der Lehrer*innenarbeit sowie ein persönliches Zeitmanagement hilfreich. Es ist also folgerichtig, auch angehenden Lehrer*innen die Möglichkeit zu geben, solche Kompetenzen zu erwerben.

\section{Das Arbeiten und Lernen in Werkstatt-Projekten}

Im Folgenden werden sieben Charakteristiken der Werkstattarbeit aufgeführt, wie sie für die durchgeführten Projekte wichtig sind.

\subsection{Komplexe Fragestellung}

Wie bereits erwähnt, bereiten akademische Berufe auf den Umgang mit komplexen Fragestellungen vor. Ähnlich verantwortungsvolle oder sogar noch verantwortungsvollere Berufe, wie beispielsweise Lokführer*innen oder Busfahrer*in, erfordern eine andere Ausbildung, die wesentlich stärker auf schematische und vorausplanbare Lösungen setzt. Dies kann jedoch für den Alltag der Lehrkraft mit den unterschiedlichen Anforderungen des Unterrichts in verschiedenen Jahrgangsstufen, in verschiedenen Themenbereichen oder in verschiedenen Tagesformen der Beteiligten nicht ausreichend sein.

Insofern ist eine von Rezepten und klaren Anweisungen geprägte Ausbildung nicht mit einem Studium vergleichbar und wäre für die Schule auch wenig angemessen. Das Studium muss die Verarbeitung komplizierter und unüberschaubarer 
Problemlagen beinhalten, um eine sinnvolle Vorbereitung auf den Lehrer*innenAlltag zu leisten. Hierzu sind die Anleitung und Unterstützung durch das akademische Lehrpersonal unumgänglich. Diese Kompetenzen erlangen die Studierenden nicht nur in Laboren und dort unter kontrollierten Rahmenbedingungen durchgeführten Experimenten, sondern in Räumen, die durch ihre inhaltliche und auch organisatorische Offenheit das Bearbeiten komplexer Probleme ermöglichen. Dazu können die unspezifischen Materialien einer Hochschullernwerkstatt anregen.

\subsection{Zeitlicher Rahmen}

Projekte sind immer zeitlich begrenzt. Der Zeitrahmen ist dabei sicher variabel, deckt sich im Studium aber mit dem Semesterturnus. Im beschriebenen Praktikum dauern die Projekte sechs Wochen, was einer Unterrichtseinheit im Schulalltag entspricht. Im schulischen Rahmen können Innovationsprojekte auch einmal länger dauern, wenn man etwa an die Umstellung von Curricula oder die Implementation neuer Prüfungsverfahren denkt. Unterrichtsprojekte von Schüler*innen sind jedoch meist ebenfalls auf ein Semester beschränkt.

Die zeitliche Begrenzung ist eine große Hilfe, um Projekte sinnvoll gestalten zu können. Sie hilft vor allem dagegen, sich in einer als, endlos' empfundenen Innovationssituation zu sehen. Gleichzeitig verhilft sie zu einem organisierten Zeitund Ressourcenmanagement.

\subsection{Zielfestlegung}

Die Zielorientierung von Projekten ist ebenfalls eine Stärke der Projektarbeit. Auch wenn die Zielklärung nicht immer einfach ist - insbesondere in Gruppen - kann sie doch einen wesentlichen Schritt der Projektarbeit darstellen. Die Aushandlungen im Falle von Zielkonflikten sind normale Praxis und nehmen auch im universitären Praktikum zahlreiche Aspekte professioneller Projektarbeit vorweg. Eine klare Zielfestlegung muss gelernt werden, und sie hilft immens bei der Zeitund Ressourcenplanung. Um es umgangssprachlich auszudrücken: wer kein Ziel hat, irrt orientierungslos umher. Dabei ist nach der Festlegung eine starre Strategie zur Zielerreichung wenig hilfreich. Ähnlich einer Wandergruppe, die in einem unbekannten Gelände auf dem Weg zum Tagesziel ist, müssen immer wieder kleinere oder größere Justierungen möglich sein. Störungen und Abweichungen sind üblich und lassen sich nicht vorausplanen, sondern müssen in den jeweiligen Situationen zur Korrektur des eingeschlagenen Weges evaluiert werden.

\subsection{Sichtbare Projektergebnisse}

Alle Projekte der Werkstattarbeit liefern sichtbare und umsetzbare Ergebnisse. Ein Think-Tank ist insofern keine Werkstattarbeit, auch eine Planungsgruppe für 
strategische Entscheidungen nicht. Die von Projektgruppen erstellten greifbaren Projektergebnisse lassen sich deshalb sowohl in Gegenständen, in einer Präsentation und auch in einer schriftlichen Fassung darstellen.

Dabei sind die Ergebnisse so strukturiert, dass sie als mögliche Unterrichtsmaterialien auch von anderen Teilnehmer*innen des Praktikums genutzt werden können. Sie sind mit einer Einführung in die Thematik, der Begründung der Auswahl und Arbeitsbögen oder auch Links zu verwendbaren Internet-Angeboten versehen. Wenn die Ideen bereits in der Praxis erprobt wurden, sind Filme oder Fotos vorhanden, die die Ergebnisse zeigen. Als besondere Vertiefung wurde eine biologiedidaktische Einordung und Aufarbeitung der Werkstattberichte verlangt. Hierfür wurden beispielsweise aktuelle Forschungsergebnisse aus Dissertationen oder Diskussionen mit Fachleuten zitiert und aufbereitet.

\subsection{Teamleistung und interdisziplinäres Vorgehen}

Projektarbeit bedingt Teamarbeit. Projektteams in Unternehmen werden häufig bewusst durch verschiedene Persönlichkeiten und verschieden professionell ausgerichtete Mitglieder besetzt, wodurch eine interdisziplinäre Zusammensetzung erreicht wird. Durch die Zusammenarbeit von Studierenden mit unterschiedlichem Zweitfach können unterschiedliche Kompetenzen kombiniert werden, die die Zusammenarbeit stark bereichern. Alle Studierenden arbeiten in Paaren oder Dreierteams, die eine genügend hohe Arbeitsdichte ermöglichen. Da die Teambildung einige Zeit in Anspruch nimmt, die Gruppen sich aber meist nur einmal pro Woche treffen, erlauben größere Gruppen kaum eine ausreichende Entwicklung des Teamcharakters.

Die konsequente Arbeit in Teams soll eine Vorbereitung auf die Arbeit an Schulen sein. Auch hier ist das Fachteam oder das Projektteam die Keimzelle für eine sinnvolle Arbeit. Die Stärkung der Fachschaftsarbeit ist das erklärte Ziel vieler Innovationsprojekte. Dabei spielen sowohl die Arbeitsteilung als auch die gegenseitige Rückversicherung und weitere Funktionen eines kollegialen Coachings eine wesentliche Rolle, eine Unterstützung, die Einzelkämpfer*innen nicht erleben können.

\subsection{Praxisbezug}

Bereits in der Beschreibung der Ergebnisorientierung wurde angedeutet, dass Projekte immer einen praktischen Bezug haben. Die Projekte im Praktikum sind auf die Schulpraxis orientiert. Sie ranken sich um aktuelle Themen oder auch klassische Unterrichtsinhalte, die mit einer Variation der Methoden besser erreicht werden können. Die Projekte haben also einen innovativen inhaltlichen oder auch methodischen Ansatz. Die Themenliste ermöglicht eine Abschätzung, in welche Richtung die Projekte gestaltet werden. 


\subsection{Projektmanagement}

Die klassischen Elemente eines Projektmanagements werden im Praktikum nur zum Teil umgesetzt. Dies liegt vor allem an der begrenzten Zeit und am überschaubaren Ressourcenmanagement. Von den üblichen neun Elementen eines Projektmanagements (PMI 2013) werden die ersten vier in unser Werkstattarbeit umgesetzt:

a) Klärung von Inhalt und Umfang

b) Termine

Die Termine sind durch den Semesterverlauf vorgegeben und bieten nur eine geringe Varianz.

c) Kosten

Die Kosten werden so gering wie möglich gehalten, die Übernahme von Fahrtkosten ist aber möglich, wenn es sich um Exkursionen oder die Teilnahme an Seminaren handelt.

d) Qualitätsmanagement

Die Qualität der Projektergebnisse wird durch die Bewertung der Präsentationen (mündlich und schriftlich) überprüft.

In unseren Projekten von geringerer Bedeutung sind die weiteren fünf Elemente:

e) Integration in das Unternehmen

f) Personalmanagement

g) Kommunikation des Projektfortschritts

h) Risikomanagement

i) Beschaffungsmanagement.

\section{Ablauf und Organisation des Praktikums}

Die rund 50 Teilnehmer*innen trafen sich an zwei Wochentagen, die angebotene Arbeitszeit betrug vier Stunden pro Woche. Für die Projektteams aus jeweils drei Studierenden stand die Werkstatt jedoch auch außerhalb der Seminar- bzw. Laborzeit nach Absprache zur Verfügung.

Die Themen konnten aus einer Liste ausgewählt werden. Sie umfassen sowohl Projekte, die für die Sekundarstufe I geeignet sind, als auch Oberstufenprojekte. Die Thematik ist breit gestaffelt und reicht von außerschulischen Aktivitäten bis hin zu alternativen Herangehensweisen an klassische Themen des Unterrichts, wie beispielsweise Mendel-Genetik (siehe Tabelle 1). 
Tab. 1: Themen für Projekte im Rahmen der Werkstattarbeit

1. Biologie im Freiland 1: Ökosystem Wiese

2. Biologie im Freiland 2: Wasseruntersuchungen

3. Biologie im Freiland 3: Wildnis Bildung

4. Biologie im Schülerlabor (mit Exkursion)

5. Sexualpädagogik I

Grundlagen, Verhütung, Schwangerschaft

6. Sexualpädagogik II

Sexuelle Orientierung, Was ist Liebe?

7. Biologie auf Englisch (oder Französisch)

8. Genetik in der Mittelstufe

Basics anhand humangenetischer Themen (also nicht der „olle Mendel“ mit seinen Erbsen)

9. Genetik in der Oberstufe

Inklusive Gentechnik, Gendiagnose etc.

10. Einführung gentechnischer Methoden in der Landwirtschaft:

öffentliche Akzeptanz und naturwissenschaftliches Basiswissen in der Bevölkerung (Exkursion in das Leibniz-Institut Gatersleben)

11. Mikrobiologische Versuche in der Schule

12 Verhaltenskunde

Experimente mit Tieren (Tierschutz beachten!)

13. Informationsverarbeitung im Gehirn

Lernen, Gehirnentwicklung, Sinneswahrnehmung im Gehirn

14. Drogen und Gehirn

Wie entstehen Gefühle? Wie wirkt Musik? Wie wirken Drogen?

15. Ökologie und nachhaltige Entwicklung: PRONAS

Hier geht es um Zukunftsszenarien bei verschiedenen Verläufen der Klimaentwicklung, Virtuelle Exkursionen, insgesamt ein interaktives Projekt, das bereits biologiedidaktisch beforscht wurde

16. Ökologie im Kontext: Es stinkt! (im Pferdestall)

Bodenökologie, Gülleproblematik, Ernährung der Pflanzen, Kreisläufe, etc.

Geeignet für Sek I und II, fächerübergreifend zur Chemie und Geographie

17. Das schwarze T-Shirt

Ein fächerübergreifendes Projekt zur Geographie, Chemie und Gesundheitserziehung sowie Bildung für nachhaltige Entwicklung (BNE), Oberstufe/Sek I

18. Vor dem Küssen Kaugummi kauen?

Fächerübergreifender Unterricht Sek I zu gesunder Ernährung, Zahnhygiene etc.

19. Evolution: Versuche und Veranschaulichung

20. MINT in der Schule: Tools für "Behinderte“ 
Während des Semesters wurden zwei umfangreiche Projekte und ein weiteres, auf Experimenten basierendes Kurzprojekt vorbereitet und präsentiert. Einige Gruppen entschieden sich für eine weitgehend vom Praktikumsraum unabhängige Gestaltung des Projektes. Sie besuchten einen Bauernhof, die Zoos in Halle und Leipzig, ein Seminar in Karlsruhe und eine Forschungseinrichtung oder auch Exkursionspunkte in der Umgebung der Universität. Die Projekte wurden sowohl mündlich als auch schriftlich präsentiert. Alle Ausarbeitungen sollten neben dem Unterrichtsmaterial auch eine Reflexion umfassen, die aktuelle biologiedidaktische Erkenntnisse einbezieht. Diese Dokumente wurden in einen Sammelordner im StudIP eingestellt. Die mündliche Präsentation fand in der Präsenszeit in aufeinanderfolgenden Präsentationen statt. Dadurch wurden pro Kurs vier bis sechs Projekte vorgestellt. Die Präsentationen wurden häufig durch Videoaufzeichnungen oder Fotos illustriert.

\section{Ergebnisse aus der Werkstattarbeit}

\subsection{Ein produktorientiertes Gruppenpuzzle im Kontext einer humangenetischen Beratungsstelle}

Eine humangenetische Beratungsstelle sucht Paare auf, in deren Familiengeschichte schwere Krankheiten auftraten, die vermutlich oder nachweislich auf erbbedingte Veränderungen zurückgehen. Solche Krankheiten wie Chorea huntington, Cystische Fibrose oder Kurzfingrigkeit sind zwar nicht häufig, eine sinnvolle Abschätzung des Risikos ist jedoch angemessen und verbindet im Biologieunterricht genetische Grundlagenkenntnisse mit einer anspruchsvollen Anwendung und einem für Jugendliche interessanten Kontext. Mit der Methode des von den Studierenden in der Werkstattarbeit entwickelten Gruppenpuzzles wird hier eine noch nicht sehr weit verbreitete Methode der Informationsaufarbeitung erprobt. Schüler*innen fällt es oft schwer, Fach- und Alltagssprache situations- und adressatengerecht anzuwenden. Im Unterricht sollen die Lernenden für diese Sprachebenen sensibilisiert und deren jeweilige Funktionen verdeutlicht werden. In diesem Projekt leiten handlungs- und schülerorientierte Aufgaben zur angemessenen Nutzung von Fach- und Alltagssprache in mündlichen und schriftlichen Kommunikationssituationen an. Den thematischen Rahmen liefert eine humangenetische Beratungsstelle.

Die Durchführung dieses Projektes lässt sich in zwei Phasen einteilen. In der ersten Phase liegt der Schwerpunkt auf der Förderung der adressaten- und situationsgerechten Benutzung von Fach- und Alltagssprache. Die Schüler*innen erarbeiten selbstständig Informationen aus einem bereitgestellten Materialangebot und erstellen als schriftliches Arbeitsergebnis ein Informationsblatt. In der zweiten 
Phase werden aus den Expert*innengruppen „Berater*innenteams“ gebildet, die in Form eines handlungsorientierten Rollenspiels eine genetische Beratung mit einem „Patient*innenpaar“ durchführen.

\section{Aufarbeitung des Themas}

Entwerft in der Gruppe ein Informationsblatt (DIN A4), das als Vorabinformation für junge schwangere Paare dienen soll, bei denen der Verdacht besteht, dass einer der/die Partner*in zumindest Überträger*in einer genetisch bedingten Krankheit ist.

Beachtet bei der Zusammenstellung des Informationsblattes folgende Kriterien: Gestaltet das Informationsblatt informativ, strukturiert und ansprechend.

Die Broschüre sollte so allgemeinverständlich geschrieben sein, dass sie unabhängig vom Bildungsstand der Patient*innen verstanden werden kann. Ihr solltet also unnötiges Fachvokabular, lange Schachtelsätze und komplexe Abbildungen vermeiden.

Gebt zunächst eine allgemeine Antwort auf die Frage, was eine genetisch bedingte Krankheit ist. Liefert dann Informationen über die genetisch bedingte Krankheit, mit der ihr euch genauer beschäftigt habt. Zeigt Möglichkeiten auf, diese Krankheit bei einem ungeborenen Kind nachzuweisen (Pränataldiagnostik).

Nehmt keine Bewertung der Situation vor. Beeinflusst werdende Eltern, die Rat suchen, nicht in ihrer Entscheidung.

Die Evaluation des Informationsblattes erfolgt mit einem Fragebogen, den die Studierenden in Anlehnung an fachdidaktische Lehrbücher gestaltet haben:

Unverständlich oder gut erklärt?

\section{Arbeitsaufträge:}

1. Lies das Informationsblatt sorgfältig durch.

2. Markiere die biologischen Fachbegriffe, die für das Verständnis der Krankheit wichtig sind.

3. Kennzeichne die Fachbegriffe, die im Informationsblatt gut verständlich erklärt werden, mit einer durchgehenden Unterstreichung, solche, die eher schwer verständlich sind, mit einer gestrichelten Unterstreichung und nicht erklärte Begriffe durch Einklammern ( ).

4. Beurteile die Gestaltung der Informationsfaltblätter anhand des folgenden Kriterienkatalogs: 


\begin{tabular}{|c|c|c|c|c|}
\hline & trifft zu & trifft eher zu & trifft weniger zu & trifft nicht zu \\
\hline fachliche Korrektheit & $\square$ & $\square$ & $\square$ & $\square$ \\
\hline $\begin{array}{l}\text { angemessener } \\
\text { Informationsgehalt }\end{array}$ & $\square$ & $\square$ & $\square$ & $\square$ \\
\hline klare Struktur & $\square$ & $\square$ & $\square$ & $\square$ \\
\hline $\begin{array}{l}\text { ansprechende } \\
\text { Gestaltung }\end{array}$ & $\square$ & $\square$ & $\square$ & $\square$ \\
\hline $\begin{array}{l}\text { Verständlichkeit für } \\
\text { die Zielgruppe in } \\
\text { Bezug auf Sprach- } \\
\text { wahl }\end{array}$ & $\square$ & $\square$ & $\square$ & $\square$ \\
\hline $\begin{array}{l}\text { Komplexität und } \\
\text { Länge der Texte }\end{array}$ & $\square$ & $\square$ & $\square$ & $\square$ \\
\hline $\begin{array}{l}\text { enthält allgemeine } \\
\text { Informationen zu } \\
\text { genetisch bedingten } \\
\text { Krankheiten }\end{array}$ & $\square$ & $\square$ & $\square$ & $\square$ \\
\hline $\begin{array}{l}\text { eine Beschreibung } \\
\text { der Erbkrankheit } \\
\text { im Speziellen und } \\
\text { Möglichkeiten zur } \\
\text { Pränataldiagnostik }\end{array}$ & $\square$ & $\square$ & $\square$ & $\square$ \\
\hline $\begin{array}{l}\text { enthält keine } \\
\text { Bewertung der } \\
\text { Handlungsoption }\end{array}$ & $\square$ & $\square$ & $\square$ & $\square$ \\
\hline
\end{tabular}

In ähnlicher Weise wird auch das Beratungsgespräch evaluiert und ein entsprechendes Feed-Back entwickelt.

Insgesamt zeigt die Einheit eine gute Verbindung von biologischen Themen mit dem Kompetenzbereich Kommunikation. Besonders aber wird auch der Kompetenzbereich Bewertung einbezogen, der nach unseren Beobachtungen im Biologieunterricht noch stark unterrepräsentiert ist.

\subsection{Vor dem Küssen Kaugummi kauen? Eine kontextbasierte Einheit zur Mundhygiene}

Dieses Projekt liefert ein gutes Beispiel für die Werkstattarbeit: im Materialangebot der Werkstatt finden sich zahlreiche Anregungen für Unterrichtsverläufe, Modelle von Zähnen mit fortschreitender Karies, Internet und Laptops, und im benachbarten Labor sind die Versuchsmaterialien für die chemischen Versuche 
vorhanden. Wie die Studierenden dieses Angebot umsetzen, bleibt ihnen überlassen.

\section{Anlass}

In Deutschland leiden etwa 98\% der Bevölkerung an Karies. Etwa 20\% der 16-Jährigen leiden an massiven Zahnverstümmelungen. Diese Ergebnisse zeigen, dass die Prävention bei Kindern und Jugendlichen nur unzureichend ist und unbedingt verbessert werden muss. Um ein nachhaltiges Wissen und Handeln der Schüler*innen zu erreichen, müssen neben dem Zahnarzt und den Eltern auch die Schule als Ort der Prophylaxe in Aktion treten. Die Projektarbeit im Schulalltag ermöglicht die Schaffung eines Lernumfeldes, das die Lernenden kognitiv, motorisch und emotional anspricht und somit Lernsituationen schafft, die zum Weiterlernen anregen und ein nachhaltiges Verhalten der Schüler*innen bewirken.

\section{Ablaufplan}

Für die Durchführung des Projektes werden 8 bis 10 Unterrichtsstunden benötigt. In der ersten Stunde erfolgt die Vorstellung des Projektes durch den/die Lehrer*in. Anschließend erfolgt ein Brainstorming zum Thema „Vor dem Küssen Kaugummi kauen - Zahn- und Mundhygiene“. In Gruppen von vier bis fünf Personen erstellen die Schüler*innen eine Wortsammlung. Die Begriffe sollen zusätzlich systematisiert werden (z. B. Mind Map). Im letzten Teil der Stunde wird ein Video aus der WDR-Sendereihe Quarks \& Co gezeigt.

In der zweiten Unterrichtsstunde erhalten die Schüler*innen die Aufgabe, in Gruppen (max. vier Personen) Fragestellungen und Hypothesen zu entwickeln, an denen sie während des Projektes arbeiten möchten. Die Stoffsammlung und die Planung von Experimenten oder der Bau von Modellen sollen selbstständig geplant werden. Der/die Lehrer*in stellt Material zur Verfügung und steht den Lernenden als Berater*in zur Seite. Am Ende der Stunde muss jede Gruppe ein Thema bzw. eine Fragestellung zum Themenkomplex „Vor dem Küssen Kaugummi kauen - Zahn- und Mundhygiene“ formuliert und einen „Fahrplan“ für die Arbeitsphase entwickelt haben. Der/die Lehrer*in nimmt die Wünsche für benötigte Materialien entgegen und versucht diese bis zur nächsten Unterrichtsstunde vorzubereiten.

Die 3. und 4. Unterrichtsstunde dienen der Durchführung von Experimenten, Erstellung von Materialien oder dem Bau von Modellen. Die einzelnen Arbeitsschritte werden innerhalb der Gruppen dokumentiert (Fotos, Filme) bzw. protokolliert. 


\section{Kontextorientierter Unterricht}

Themen des Biologieunterrichts sollen mit dem Vorwissen der Schüler*innen und ihren Alltagserfahrungen verbunden werden; weiterhin soll die Verknüpfung von Lebenswelt und Wissenschaft durch ,sinnstiftende und lebenspraktische Kontexte“, ,authentische Problemsituationen erreicht werden (BAYRHUbER et al. 2007). In diesem Projekt wird das Alltagsthema „Zähne und Zahnhygiene“ mit wissenschaftlichen Experimenten verknüpft. Weiterhin spielen auch authentische Problemsituationen eine Rolle, die die Kinder und Jugendlichen in diesem Alter beschäftigen, so z. B. die Frage: Muss man vor dem Küssen Kaugummi kauen und welchen Effekt hat das?

\section{Beispiele für Experimente}

- Zahnpasta selbst herstellen

- Vergleich selbst hergestellter mit gekaufter Zahnpasta durch Untersuchung der Faktoren:

- Schleifwirkung

- pH-Wert

- Schutzwirkung gegen Säuren

- Messung des pH-Wertes im Mund nach Essen und anschließendem

- Kaugummi-Kauen

- Entfernung von Plaque durch Zähneputzen

- Kultur von Mundbakterien auf selbst hergestellten Agar-Platten

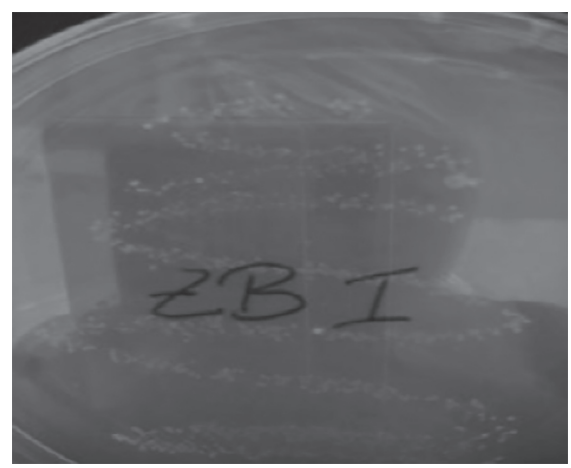

Abb. 1: Mundbakterien (kleine weiße Punkte) auf einer Agar-Platte 

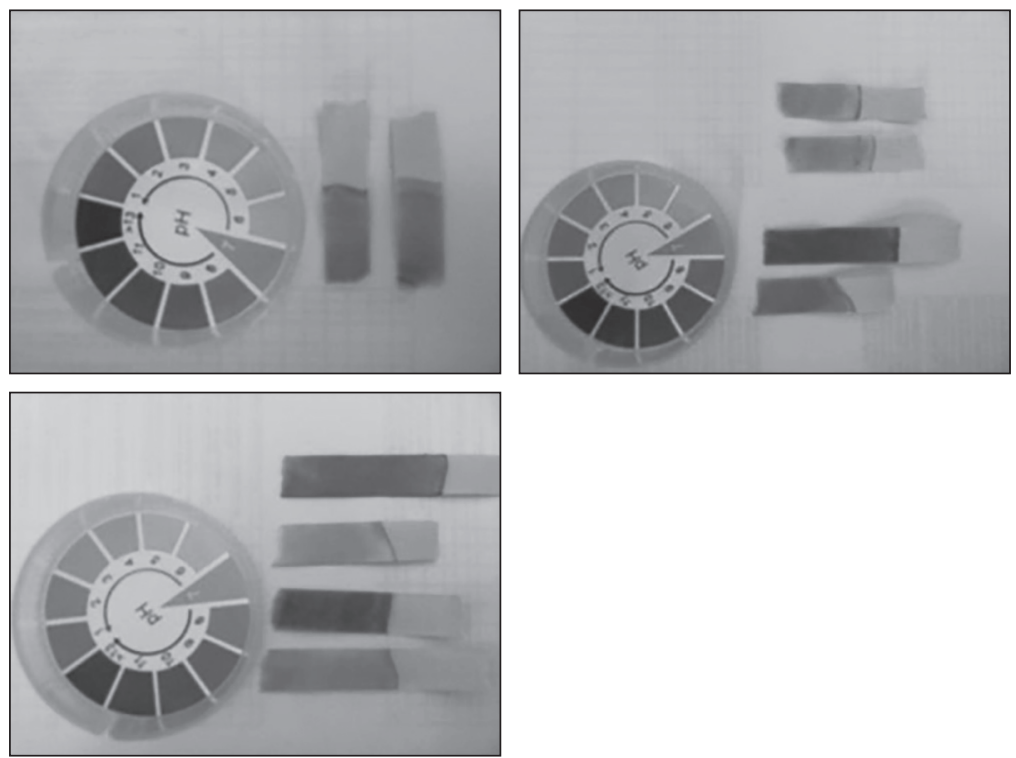

Abb. 2: pH-Wert im Mund vor dem Essen (links), nach dem Essen (Mitte) und nach dem Kaugummikauen: Der in der Werbung verbreitete Effekt, dass Kaugummikauen den pH-Wert in der Mundhöhle positiv verändert, konnte nicht erbracht werden.

\section{Auswertung der Erfahrungen der Werkstattarbeit}

In der letzten Veranstaltung wurden die Studierenden nach ihren Erfahrungen befragt. Von den insgesamt 49 Teilnehmer*innen wurden 42 Fragebögen ausgefüllt, was einer Rücklaufquote von $86 \%$ entspricht. Die Fragebögen enthielten 40 Fragen, die sich auf die im Folgenden zusammengestellten Themenkomplexe bezogen. 


\subsection{Einschätzung der Entwicklung der eigenen Kompetenzen}

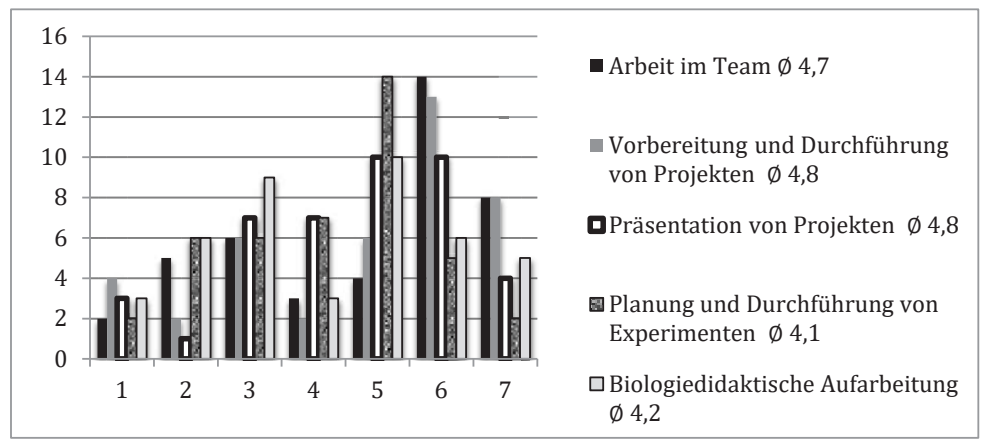

Abb. 3: Entwicklung der eigenen Kompetenzen (Selbsteinschätzung). Angegeben sind die Zahl der Nennungen ( $\mathrm{n}=42)$. 1: Ablehnung (,trifft gar nicht auf mich zu“), 7: Zustimmung (,trifft vollkommen zu")

Die Entwicklung der eigenen Kompetenzen wird leicht positiv beurteilt. Im Vergleich zur Projektarbeit und deren Präsentation (Mittelwerte 4,8) sind jedoch die experimentellen Kompetenzen etwas weniger stark gefördert worden (Mittelwert 4,1). Auch die Kompetenz zur Aufarbeitung nach didaktischen Gesichtspunkten wird mit mittlerer Antworthäufigkeit gesehen.

\subsection{Vorerfahrung, Anleitung und Zielfindung}

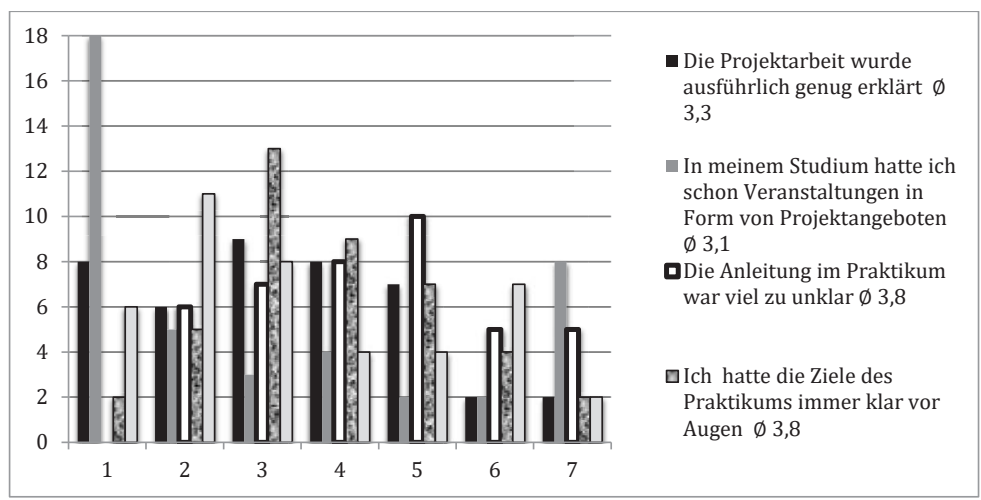

Abb. 4: Anleitung und Zielfindung. Angegeben sind die Zahl der Nennungen ( $\mathrm{n}=42) .1$ : Ablehnung („trifft gar nicht auf mich zu“), 7: Zustimmung („trifft vollkommen zu“)

Die Angaben zur Vorerfahrung zeigen, dass für die meisten Teilnehmer*innen diese Form des Praktikums neu war. Die Einführung wurde von den meisten 
als ausreichend betrachtet und auch die Zielfindung war kein größeres Problem. Dennoch war es nicht immer einfach, die gesetzten Ziele im Auge zu behalten.

\subsection{Zusammenarbeit im Team}

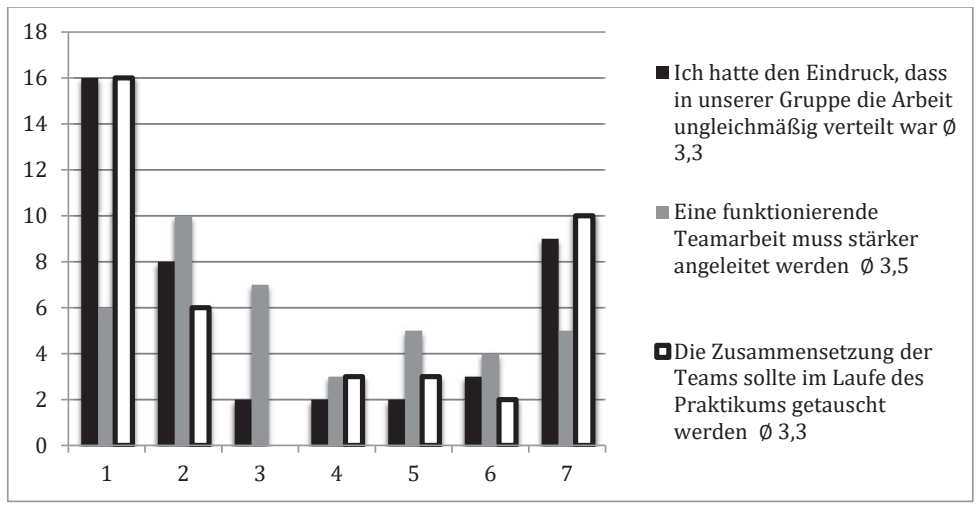

Abb. 5: Zusammenarbeit im Team. Angegeben sind die Zahl der Nennungen ( $n=42)$. 1: Ablehnung (,trifft gar nicht auf mich zu“), 7: Zustimmung („trifft vollkommen zu“)

Die Teamarbeit ist ein erklärtes Ziel des Praktikums. Umso wichtiger ist es, deren Erfolg zu evaluieren. In den meisten Gruppen funktionierte die Teamarbeit gut. In drei Teams gab es Probleme mit der Zuverlässigkeit einzelner Teilnehmer*innen, und in zwei Teams fielen jeweils einzelne Mitglieder aus. Dies drückt sich im Antwortverhalten aus: 9 bzw. 10 Studierende erlebten die Teamarbeit als eine ungleichmäßige Verteilung der Arbeit oder wünschen sich einen Wechsel in der Projektgruppe. Dies sind immerhin fast $1 / 4$ der Kursteilnehmer*innen. 


\subsection{Zeitmanagement}

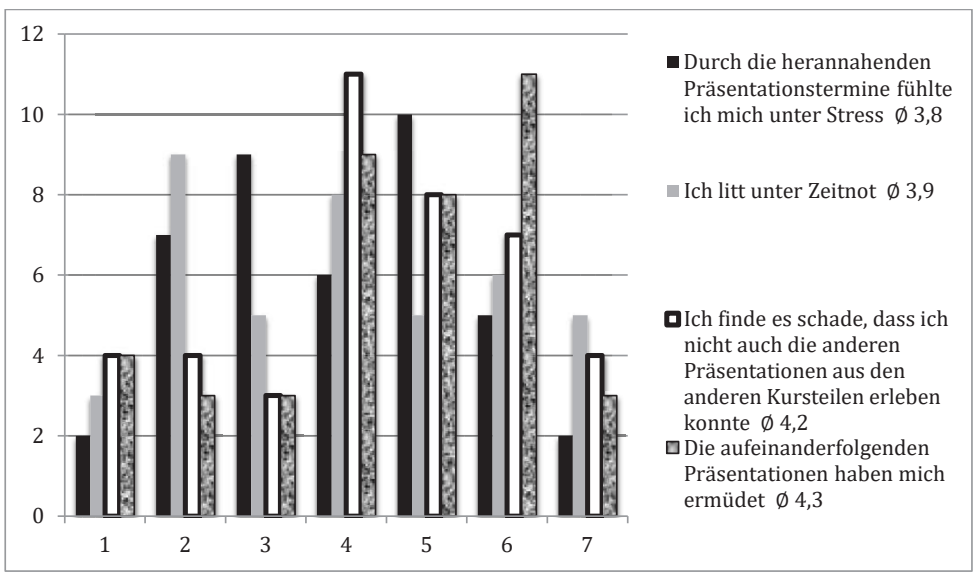

Abb. 6: Zeitmanagement. Angegeben sind die Zahl der Nennungen ( $\mathrm{n}=42)$. 1: Ablehnung (,trifft gar nicht auf mich zu“), 7: Zustimmung (,trifft vollkommen zu“)

Das Zeitmanagement ist weitgehend gelungen. Die Studierenden fühlten sich überwiegend zeitlich nicht unter Stress, sie waren von der Reihe der Präsentationen jedoch etwas ermüdet.

\subsection{Generelle Zustimmung zum Praktikum}

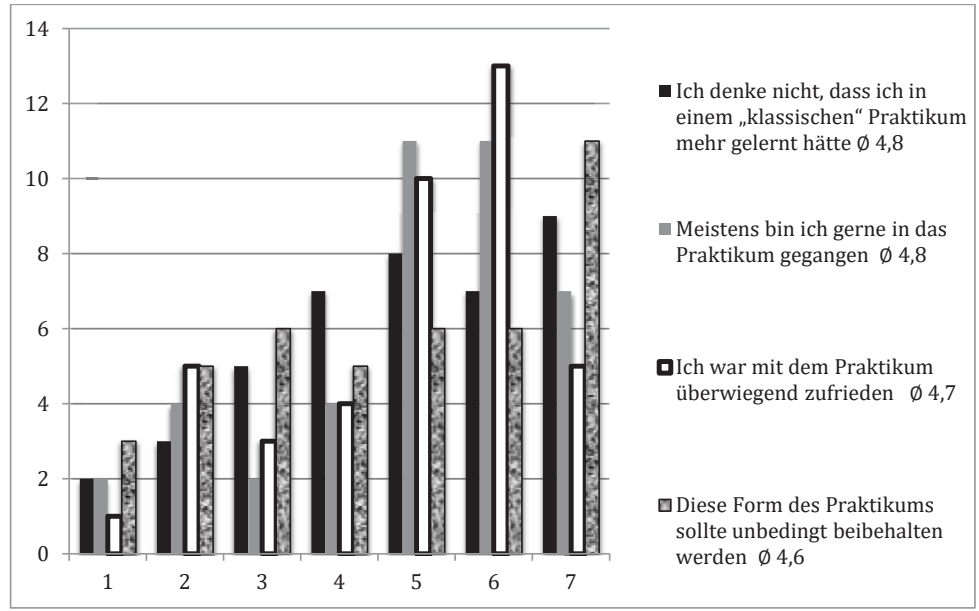

Abb. 7: Einstellung zum Praktikum. Angegeben sind die Zahl der Nennungen ( $\mathrm{n}=42)$. 1: Ablehnung (,trifft gar nicht auf mich zu“), 7: Zustimmung („trifft vollkommen zu“) 
Abschließend werden die Antworten auf die Fragen wiedergegeben, die die $\mathrm{Zu}$ stimmung oder Ablehnung zu dieser - für die Studierenden neuen - Form des Praktikums umfassen. Insgesamt zeigt sich eine Zustimmung, und auch wenn die Antworten nicht in der höchsten Zustimmungskategorie kumulieren, sind doch die beiden Antwortmöglichkeiten 5 und 6 deutlich häufiger genannt als die ablehnenden Kategorien.

\subsection{Rückmeldung zum Lehrpersonal und zum Lernangebot}

Für die Veranstalter*innen war es wichtig, eine Rückmeldung auf die Anleitung und Begleitung im Praktikum zu erhalten. Sie fiel insgesamt positiv aus (Mittelwerte über 4). Dies bezog sich sowohl auf die Unterstützung bei der Materialversorgung als auch auf das Feedback zu den Präsentationen und den Versuchen, die vorgeführt wurden. Allerdings konnten die Studierenden keine Verbindung zum Einführungspraktikum für Schulversuche erkennen. Dies kann daran liegen, dass sie an früheren Praktika teilgenommen hatten, die keine Ausrichtung auf das Projektpraktikum hatten.

Die erarbeiteten Materialien scheinen für die meisten Studierenden interessant und für den Einsatz im Unterricht geeignet zu sein. In einer nicht in der Grafik abgebildeten Antwortskala wird deutlich, dass das Material bisher noch nicht im Unterricht (z. B. in den schulpraktischen Übungen) eingesetzt wurde. Zum Experimentalanteil war die Meinung geteilt. Einige Studierende können sich durchaus einen höheren experimentellen Anteil vorstellen, andere haben jedoch auch bereits in dieser Form des Praktikums genug experimentiert. Grundsätzlich werden Experimente jedoch favorisiert.

\section{Zusammenfassung und Ausblick}

Die Umstellung des klassischen ,Kochbuchpraktikums' auf die Werkstattarbeit kann aus unserer Sicht als voller Erfolg gewertet werden. In allen abgefragten Bereichen - Zusammenarbeit, Möglichkeiten zum Experimentieren, Unterstützung durch das Lehrpersonal, Zeitmanagement, Organisation und Vorbereitung - wurden positive Rückmeldungen gegeben. Auch aus Sicht der Betreuer*innen lief das Praktikum gut. In Zukunft soll jedoch noch mehr Wert auf die fachdidaktisch fundierte Aufarbeitung gelegt werden. Ein Praxistest, etwa im Rahmen von Projektwochen an Schulen, im Rahmen der Kinderuni oder anderer außerschulischer Vorhaben von externen Trägern, ist zukünftig vorgesehen. Auch besteht die Idee, mehr und mehr Kinder und Jugendliche in die Werkstatt einzuladen und dort bei der Durchführung der Projekte zu beobachten. 
Als Erfolg kann auch gewertet werden, dass einige der Studierenden das in der Werkstattarbeit erarbeitete Thema als Staatsexamensarbeit vertiefen.

\section{Literatur}

Bayrhuber, Horst et al. (2007): Biologie im Kontext. Der mathematische und naturwissenschaftliche Unterricht. 60(5), 282-286.

Fischler, Helmut; Gebhard, Ulich \& Rehm, Markus (2018): Naturwissenschaftliche Bildung und Scientific Literacy. In: Krüger, Dirk; Parchmann, Ilka \& Schecker, Horst (Hrsg.): Theorien in der naturwissenschaftsdidaktischen Forschung. Berlin, Heidelberg: Springer-Verlag, 11-29.

Helmke, Andreas (2017): Unterrichtsqualität und Lehrerprofessionalität. Diagnose, Evaluation und Verbesserung des Unterrichts. 7. überarbeitete Auflage. Seelze: Klett-Kallmeyer.

KMK (Sekretariat der Ständigen Konferenz der Kultusminister der Länder in der Bundesrepublik Deutschland) 2004: Standards für die Lehrerbildung: Bildungswissenschaften.

LindNer, Martin (2008): New programmes for teachers' professional development in Germany. The programme SINUS as a model for teachers' professional development. Im Internet: INTERACÇÓES 9, 149-155. Im Internet: http://revistas.rcaap.pt/interaccoes/article/view/365/320.

PMI (Project Management Institute, Inc.) (2013): A Guide to the Project Management Body of Knowledge. 5. Auflage. PMI, Newtown Square PA (USA). 